\title{
Primary cilia: putting a sensor on the underlying melanocytic tumor cell state
}

\author{
Ursula E Lang ${ }^{1,2}$ \\ ${ }^{1}$ Department of Pathology, Dermatopathology Service, University of California - San Francisco, San Francisco, CA 94115, USA \\ ${ }^{2}$ Department of Pathology, Zuckerberg San Francisco General Hospital - San Francisco, San Francisco, CA 94110, USA \\ *Author for correspondence: Ursula.Lang@ucsf.edu
}

"In this study, a comprehensive review of histopathologic features, array comparative genomic hybridization $(\mathrm{aCGH})$ and ciliation index were evaluated, and a low ciliation index was ranked as one of the top features in reaching a malignant diagnosis."

First draft submitted: 8 May 2020; Accepted for publication: 28 May 2020; Published online: 17 July 2020

Keywords: ambiguous melanocytic neoplasm • diagnostic tool • melanoma • primary cilia • primary cilium

Melanocytic neoplasms constitute some of the most challenging histopathologic entities in pathology and trigger high levels of diagnostic uncertainty among general practice pathologists and even among expert dermatopathologists $[1,2]$. When histopathologic findings are ambiguous or indeterminate, one turns to immunohistochemical and/or genetic analyses, given sufficient tissue and resources [3]. These additional tests can provide valuable information about the underlying genetic drivers, level of genomic instability and relative levels of key transcripts, which are then integrated with histopathology and clinical information [4]. When fortunate enough to obtain the genetic data, the hope is for a compelling basis to definitively classify the tumor. Analysis may be less than straightforward, as mutations or chromosomal changes of unknown significance may be uncovered. Additional impediments to diagnosis include poor quality DNA, low purity of tumor cells and a chromosomal copy-number neutral melanoma. For these reasons, the gold standard remains histopathologic review by an experienced dermatopathologist.

Often the true malignant potential remains a mystery, and management is aimed at balancing caution with morbidity. In such instances, one can wonder if microRNA (miRNA), transcriptomic or DNA methylation data would shed more light onto the underlying biologic potential [5-7]. In the case of miRNAs, which are stable in formalin-fixed paraffin-embedded (FFPE) tissue, several studies have shown the potential for use as diagnostic biomarkers $[6,8]$. Although mRNA is less stable than miRNAs in FFPE, commercially available kits leverage a limited panel of gene transcripts that can predict risk of recurrence, metastasis and assist in diagnosis [9]. DNA methylation shares the advantage of stability similar to miRNAs, and appears to correlate with poor prognosis in advanced melanoma [5]. The epigenetic information gained from methylation analysis remains unknown in histopathologically indeterminate lesions. Challenges in using these tests include the lack of high-quality evidence guiding best practice use [10]. As any cell biologist will attest, it is the proteome and interactome of cells that ultimately results in behavior. While the complexity can be overwhelming, it remains a fascination and continues to propel the field of melanoma biology. It is in this vein that the assessment of primary cilia has emerged as a window into melanoma cell biology.

The primary cilium is ubiquitous cell surface organelle that acts as cellular antenna, sensing the extracellular environment and transmitting downstream signals [11]. Many important signaling pathways have been linked to the primary cilium in a cell-context-dependent manner and implicated in cancer pathogenesis [12]. With respect to melanocytes and melanoma progression, there are limited but intriguing data to support the WNT/ $\beta$-catenin pathway being repressed by an intact primary cilium [13]. Additionally, the same study proposed that deconstruction of primary cilia was in part controlled by EZH2 overexpression leading to downstream repression of essential cilia genes. EZH2 is the catalytic subunit to PRC2, and when its activity is enhanced over that of the SWI/SNF pathway, it favors malignant transformation [7]. EZH2 has been found to be overexpressed in melanoma compared with nevi [14], which would appear to be one mechanism explaining the absence of primary cilia in conventional 
melanomas. If the presence or absence of primary cilia can be linked to genomic changes that influence downstream signaling, this may represent a morphologic advance with the power to reveal the malignant potential of ambiguous melanocytic tumors.

Unequivocal conventional melanoma shows almost complete loss of primary cilia, whereas melanocytic nevi show significant retention [15-17]. As a diagnostic tool, primary cilia staining would appear to provide redundant information when the pathologic diagnosis is undisputed. In the setting of 'dysplastic nevi', the decision to re-excise is critical to management and is heavily influenced by the final histopathological diagnosis [18]. The ciliation index (percent of melanocytes containing a primary cilia) in mildly dysplastic nevi was found to be significantly higher than in that of severely dysplastic nevi (average 56 vs 14\%, respectively), suggesting a means for an objective measure to aid in management [19]. Furthermore, the utility of ciliation index as an adjunct molecular tool has been demonstrated in histopathologically ambiguous 'spitzoid' melanocytic neoplasms [20]. In this study, a comprehensive review of histopathologic features, array comparative genomic hybridization $(\mathrm{aCGH})$ and ciliation index were evaluated, and a low ciliation index was ranked as one of the top features in reaching a malignant diagnosis. The ciliation index was found to contribute independent and additive information, in the presence or absence of aCGH data. These findings, together with the relative ease of performing this stain on a single standard-thickness slide of formalin-fixed paraffin embedded tissue, make this an appealing option for use in daily clinical practice. It remains to be seen if the presence or absence of primary cilia correlates with clinical outcomes; this could be achieved through a large prospective, randomized, multi-institutional study. Until that is feasible, additional retrospective studies comparing ciliation index with the previously mentioned adjunct molecular tools and long-term clinical outcomes would be a good start.

Beyond melanoma-focused analysis, aspects of the foundational knowledge of primary cilia structure and function can be postulated in melanocytic neoplasms. Ciliogenesis is intricately connected to the cell cycle, is influenced by changes to the cytoskeleton, cellular proteostasis (i.e., autophagy) and signal transduction [11]. The mother centriole is an essential anchor point of primary cilia formation, and during cell division must be made available for mitotic spindle formation. In other words, ciliary disassembly is a prerequisite for cell-cycle re-entry. If one considers this in the context of conventional assessment of melanocytic neoplasms, primary cilia staining should be complementary to Ki-67 for evaluation of cell proliferation or the lack thereof. However, a lack of ciliation does not necessarily equate to a proliferative cell state [17]. Overall, there continues to be a need for exploration into the significance of primary cilia as a tumor suppressor and definition of the specific settings in which assessment of ciliation can be leveraged as a biomarker for clinical use.

\section{Acknowledgments}

T McCalmont was very generous with helpful suggestions and insightful discussion.

Financial \& competing interests disclosure

U Lang was supported by the Dermatology Foundation through a Dermatopathology Career Development Award. The author has no other relevant affiliations or financial involvement with any organization or entity with a financial interest in or financial conflict with the subject matter or materials discussed in the manuscript apart from those disclosed.

No writing assistance was utilized in the production of this manuscript.

\section{Open access}

This work is licensed under the Attribution-NonCommercial-NoDerivatives 4.0 Unported License. To view a copy of this license, visit http://creativecommons.org/licenses/by-nc-nd/4.0/

\section{References}

1. Piepkorn MW, Longton GM, Reisch LM et al. Assessment of second-opinion strategies for diagnoses of cutaneous melanocytic lesions. JAMA Netw. Open 2(10), e1912597 (2019).

2. Elmore JG, Barnhill RL, Elder DE et al. Pathologists' diagnosis of invasive melanoma and melanocytic proliferations: observer accuracy and reproducibility study. BMJ 357, j2813 (2017).

3. Lang UE, Yeh I, McCalmont TH. Molecular melanoma diagnosis update: gene fusion, genomic hybridization, and massively parallel short-read sequencing. Clin. Lab. Med. 37(3), 473-484 (2017).

4. Miedema J, Andea AA. Through the looking glass and what you find there: making sense of comparative genomic hybridization and fluorescence in situ hybridization for melanoma diagnosis. Mod. Pathol. doi:10.1038/s41379-020-0490-7 (2020) (Epub ahead of print). 
5. Yamamoto Y, Matsusaka K, Fukuyo M, Rahmutulla B, Matsue H, Kaneda A. Higher methylation subtype of malignant melanoma and its correlation with thicker progression and worse prognosis. Cancer Med. doi:10.1002/cam4.3127 (2020) (Epub ahead of print).

6. Torres R, Lang UE, Hejna M et al. MicroRNA ratios distinguish melanomas from nevi. J. Invest. Dermatol. 140(1), 164.e7-173.e7 (2019).

7. Shain AH, Joseph NM, Yu R et al. Genomic and transcriptomic analysis reveals incremental disruption of key signaling pathways during melanoma evolution. Cancer Cell 34(1), 45.e4-55.e4 (2018).

8. Quiohilag K, Caie P, Oniscu A, Brenn T, Harrison D. The differential expression of micro-RNAs 21, 200c, 204, 205, and 211 in benign, dysplastic and malignant melanocytic lesions and critical evaluation of their role as diagnostic biomarkers. Virchows Arch. doi:10.1007/s00428-020-02817-5 (2020) (Epub ahead of print).

9. Yang K, Oak ASW, Slominski RM, Brożyna AA, Slominski AT. Current molecular markers of melanoma and treatment targets. Int. J. Mol. Sci. 21(10), 3535 (2020).

10. Grossman D, Kim CC, Hartman RI et al. Prognostic gene expression profiling in melanoma: necessary steps to incorporate into clinical practice. Melanoma Manag. 6(4), MMT32 (2020).

11. Malicki JJ, Johnson CA. The cilium: cellular antenna and central processing unit. Trends Cell Biol. 27(2), 126-140 (2017).

12. Choudhury A, Neumann NM, Raleigh DR, Lang UE. Clinical implications of primary cilia in skin cancer. Dermatol Ther (Heidelb). 10(2), 233-248 (2020).

13. Jackson PK. EZH2 inactivates primary cilia to activate Wnt and drive melanoma. Cancer Cell 34(1), 3-5 (2018).

14. McHugh JB, Fullen DR, Ma L, Kleer CG, Su LD. Expression of polycomb group protein EZH2 in nevi and melanoma. J. Cutan Pathol. 34(8), 597-600 (2007).

15. Lang UE, Love NR, Cheung C, McCalmont TH, Kim J. Use of the ciliation index to distinguish invasive melanoma from associated conventional melanocytic nevi. Am. J. Dermatopathol. 42(1), 11-15 (2019).

16. Kim J, Dabiri S, Seeley ES. Primary cilium depletion typifies cutaneous melanoma in situ and malignant melanoma. PLoS ONE 6(11), e27410 (2011).

17. Snedecor ER, Sung CC, Moncayo A et al. Loss of primary cilia in melanoma cells is likely independent of proliferation and cell cycle progression. J. Invest. Dermatol. 135(5), 1456-1458 (2015).

18. Kim CC, Berry EG, Marchetti MA et al. Risk of subsequent cutaneous melanoma in moderately dysplastic nevi excisionally biopsied but with positive histologic margins. JAMA Dermatol. 154(12), 1401-1408 (2018).

19. Lang UE, Cheung C, Vladar EK, Swetter SM, Kim J. Loss of primary cilia correlates with cytologic severity in dysplastic melanocytic nevi: loss of primary cilia correlates with cytologic severity. J. Cutan. Pathol. 43(2), 113-119 (2016).

20. Lang UE, Torres $\mathrm{R}$, Cheung $\mathrm{C}$ et al. Ciliation index is a useful diagnostic tool in challenging spitzoid melanocytic neoplasms. J. Invest. Dermatol. doi:10.1016/j.jid.2019.11.028 (2020) (Epub ahead of print). 
